\title{
Polyphonie et le Roméo et Juliette de Baz Luhrmann
}

\section{Raphaëlle Costa de Beauregard}

\section{Q OpenEdition}

\section{Journals}

Édition électronique

URL : http://journals.openedition.org/shakespeare/327

DOI : 10.4000/shakespeare.327

ISSN : 2271-6424

Éditeur

Société Française Shakespeare

Édition imprimée

Date de publication : 1 novembre 1999

Pagination : 89-108

ISBN : 2-84269-331-0

Référence électronique

Raphaëlle Costa de Beauregard, «Polyphonie et le Roméo et Juliette de Baz Luhrmann », Actes des congrès de la Société française Shakespeare [En ligne], 17| 1999, mis en ligne le 01 novembre 2007, consulté le 01 mai 2019. URL : http://journals.openedition.org/shakespeare/327 ; DOI : 10.4000/ shakespeare.327 


\section{S H A K E S P E A R E \\ \& $\quad$ L $A \quad$ V O I X}

Société Française Shakespeare

Actes du Congrès de 1999

米米

Textes réunis et présentés par

Patricia DORVAL

publiés sous la direction de

Jean-Marie MAGUIN 


\section{P O L Y P H O N I E E T L E ROMÉO ET JULIETTE DE BAZ L U H R M A N N ${ }^{1}$}

Polyphonie ? Cacophonie, dirons certains, au sortir de la projection de ce film ! Et pourtant la critique a dans l'ensemble été plutôt favorable à cette nouvelle adaptation de la tragédie de Shakespeare à l'écran ${ }^{2}$, et le succès fut immense aux États-Unis ${ }^{3}$. Une première difficulté à prendre ce film au sérieux est celle que rencontrent les critiques pour le cataloguer. Certains parlent de «la première tragédie MTV», ou de «psychographie» (Première, mai 1997), d'autres de «cacophonie» avec tout de même un «beau plan» (Positif, mai 1997), un «gigantesque clip MTV» (Studio, avril 1997), ou de «marasme esthétique», doublé d'une «collection de signes d'époque pour reportage de mode» (Cahiers du Cinéma, avril 1997). Le film a aussi été appelé "a visceral epic» ${ }^{4}$, suggérant qu'à un certain public c'est une impression d'unité esthétique qui se dégage, alliant action, spectacle, romanesque pour le plaisir du spectateur ${ }^{5}$.

On remarquera que le titre du film n'est pas le même pour un spectateur anglophone et un spectateur français, car pour les premiers il importe de citer le titre en entier, ce qui signifie commencer par William Shakespeare et attirer l'attention sur ce que le film prétend être : une réflexion sur l'art de Shakespeare et non la mise en scène d'une histoire d'amour. Dans l'interview citée cidessus, Luhrmann parle d'une question centrale à laquelle le film apporterait un élément de réponse : «si Shakespeare était vivant 
aujourd'hui, quel film ferait-il ?».

Par ailleurs les critiques consultés sont unanimes: le texte serait restitué intégralement. Une rapide comparaison entre la bande son du film et le texte nous assure aussitôt du contraire : très peu de vers sont utilisés dans le film, et au moins la moitié d'entre eux sont déplacés, n'apparaissant pas sur l'axe syntagmatique du film au même endroit que dans le texte de la pièce, et donc ne faisant pas référence au même contexte énonciatif, tout en respectant le contexte situationnel, c'est-à-dire paradigmatique: scène de violence, scène d'amour par exemple. D'autre part, certains vers sont répétés plusieurs fois par le personnage, scandant le discours filmique et s'imprimant dans la mémoire du spectateur à la manière du refrain d'une chanson dont on aurait oublié les strophes mais dont la signification serait néanmoins présente. Et enfin, certains vers sont remplacés par un cri, à la manière des ruptures du chant d'opéra dont la fonction est d'attirer l'attention sur la qualité de la voix entendue plutôt que sur le contenu des mots prononcés, ce que Michel Chion appelle l'écoute réduite ${ }^{6}$. On peut donc se demander si l'effet de sens /restitution intégrale/ n'est pas véhiculé par des procédés rhétoriques autres que la citation, et en particulier par le contraste entre la voix «érudite» qui sait identifier et reproduire un texte culte et le contexte où ces citations sont produites, lequel est éminemment connoté : «populaire». Ce contraste peut déplaire aux tenants de la différence entre «high culture» et «low culture», selon lesquels il existerait une différence de nature entre la «vraie culture» et la «culture de masse», mais il peut aussi fasciner ceux qui perpétuent l'idéal de la sémiotique selon lequel tout ce qui fait sens mérite d'être analysé, et le contraste entre «high» et «low» n'est qu'un effet de sens de plus qui vient simplement s'ajouter aux autres effets de sens produits par le spectateur lors de la réception de l'œuvre.

Les lignes qui suivent adoptent cette dernière approche tout en se limitant à quelques aspects seulement de cette polyphonie culturelle, à savoir : la cacophonie, l'opéra, et, peut-être, un souffle shakespearien, dans William Shakespeare's Romeo + Juliet.

\section{La cacophonie}

Chez Shakespeare, le son ne se limite pas à la musique et au bruitage, mais constitue un support spectaculaire constant. Le théâtre de Shakespeare explore les possibilités d'une véritable audio-vision où le son et l'image se complètent selon les fonctions sémantiques inventoriées par Michel Chion. Le spectateur 
shakespearien est en effet convié à une écoute réduite à chaque fois qu'un nouvel acteur entre sur scène et introduit le timbre de sa voix dans la trame sonore : cela est vrai en particulier pour le problème de la mue des voix de jeunes garçons. Mais bien entendu le timbre de la voix de l'acteur est aussitôt traduit en identité de personnage, de sorte que Viola / Cesario tire parti de son timbre de voix pour séduire Orsino alors même qu'elle doit l'utiliser pour séduire Olivia au nom du même Orsino. Il en va de même pour Rosalind / Ganymede / Rosalind. Cette valeur iconique de la voix permet des jeux sur l'identité des personnages.

Dans la pièce élisabéthaine, la première scène tire parti d'effets semblables. Le dialogue rapide des deux partisans des Capulet s'oppose à la voix unique du lecteur du prologue. Cette présentation des deux clans se fait sous le signe de la théâtralité : le double partenariat des Capulet (Sampson et Gregory) donne libre cours à des effets de manche pléthoriques, se traduisant par des sonorités se faisant écho, et non sur l'échange d'un message particulier. Ceci est souligné par l'usage de la stichomythie dans les premières répliques (1-7), puis l'allongement des répliques et de cet effet par l'utilisation de deux phrases chacune (jusqu'à la ligne 14). Le contenu joue sur la duplication des sons, par l'allitération en [k] de «carry coals», «colliers», et «neck out of collar», la répétition de «strike» $(5 \& 6)$, puis de «stand» $(8,10 \& 27)$, et la multiplication des signifiés (sens littéral, figuratif, et ordurier) qui en découle. L'effet recherché par les interlocuteurs est d'attirer l'attention sur les sons qui s'entrechoquent et se répondent d'une manière désordonnée quoique ponctuée par les répétitions. Pour le spectateur, il s'agit en outre d'un mime auriculaire d'une bataille imaginaire annonçant la bataille réelle qui va se dérouler quelques secondes plus tard, entre les clans ennemis cette fois-ci. La scène est donc caractérisée par un mimodrame reproduisant dans un miroir en miniature, entre alliés, le récit de la vendetta auquel le prologue est consacré et, d'une manière plus littérale, la bataille rangée entre les deux clans qui constitue l'armature de l'intrigue.

Après un prologue confié à une présentatrice de télévision auquel fait suite un générique présentant la ville de Fair Verona (il s'agit de Mexico City alias Los Angeles) depuis la lunette violemment chahutée d'un hélicoptère de police, le film s'ouvre sur un groupe de bruyants lurons en goguette filant à vive allure à bord de leur décapotable jaune. Celui-ci s'avère être le clan des Montague, et c'est Benvolio qui est au volant. Deux d'entre eux arborent les insignes du Punk, l'un s'étant fait tatouer le nom de 
Montague sur sa nuque rasée, et l'autre affublé d'une crête de cheveux rouges. Ce dernier pousse des cris imitant celui de bêtes sauvages, et son œil poché connote le cirque (œil poché, cheveux rouges, chemise bariolée), c'est-à-dire au sens littéral le qualificatif que l'on serait tenté d'appliquer à leur comportement à la fois débridé, voire ordurier (le jeu de mots sur «maiden» et «maidenhead», (21-5) est transposé en un geste obscène à l'intention des passantes - une religieuse et un groupe de jeunes filles en l'occurrence).

Or, pour le spectateur familier avec la pièce élisabéthaine, il y a là une inversion significative. La distinction entre les deux clans fait en effet problème : le prologue souligne le fait qu'ils sont égaux «both alike in dignity» et que par conséquent leur vendetta est absurde. Toutefois l'ordre d'apparition sur scène confère au premier clan, celui des Capulet et donc de Juliette l'avantage sur le second de dominer l'espace scénique d'entrée de jeu. La liste des personnages confirme cette prééminence en donnant aux Capulet quatre jeunes gens là où les Montague n'en ont qu'un, Abram. Par contre, s'ils sont plus nombreux, ils sont aussi moins décidés et lors de la confrontation entre Benvolio, neveu pacifiste des Montague et Tybalt, bouillant neveu des Capulet, c'est le clan des Capulet qui paraît le plus hardi et ardent au combat. Cette cacophonie est donc bien la transposition de l'ouverture de la pièce, mais l'inversion de l'ordre d'apparition des deux familles et donc d'une répartition calculée des valeurs représentées par chaque clan vient compliquer les choses. Le clan des Capulet entre en scène avec Tybalt de sorte que le clan de Juliette contraste fortement avec celui de Roméo.

La connotation d'ethnicité devient évidente et avec elle l'effet citationnel renvoyant à West Side Story. La station d'essence devient emblématique du terrain à conquérir, ce qui est renforcé par un insert non seulement sur «Phoenix gas» sur fond rouge mais aussi sur un deuxième insert : «Add more fuel to your fire - Phoenix» sur fond jaune. La connotation ironique de cette publicité devient évidente quand la station service s'embrase. La conduite intérieure bleue nuit des Capulet contraste avec la décapotable jaune, et le cadrage sur Abra, un Capulet (dans la pièce, Abram, du parti des Montague) est motivé par ce qui prétend être une réaction d'indignation au geste obscène mentionné ci-dessus. Les zooms sur leurs blasons : Montague et Capulet sur la crosse de leur pistolet, montés en syntagme alterné ${ }^{7}$ grâce à un cut - et un plan serré à la manière d'un insert - sur chacun d'eux, soulignent la rivalité des deux clans. Mais ces deux inserts fonctionnent aussi comme des 
syntagmes parallèles, chacun projetant sur l'autre une signification d'ordre symbolique : nostalgie de la dimension épique de glorieux combats ancestraux, soif frénétique de vengeance et de pouvoir, viennent aussitôt à l'esprit.

Le conflit ethnique se double dès lors d'un conflit culturel : les Hispanos (ou Latinos) sont bruns et barbus, mais aussi tatoués (dos d'Abra) ou ceinturés d'icônes de la Vierge, quand ceux-ci ne sont pas brodés sur un gilet porté à même la peau nue et ajusté à la manière d'un corset (Tybalt); leurs opposants sont «roux» et arborent des chemises Hawaïennes multicolores flottant au vent et une nudité provocante. Le blason or et bleu sur fond gris argent pour les Montague constitue un reflet inversé de celui des Capulet - or et rouge sur fond gris argent. Ces différences comportent donc un dénominateur commun qui suggère non seulement la différence de culture mais aussi le redoublement du même. Certes leur bataille se déroule en pleine rue au mépris des autres voitures, et son caractère grotesque est rehaussé par un épisode clownesque lorsque la passagère hystérique d'une autre voiture s'en mêle à l'aide d'un sac qu'elle manie comme un gant de boxe sans pour autant obtenir de résultat; et pourtant leur affrontement est réglé comme une chorégraphie de comédie musicale.

Le film utilise donc l'effet de dédoublement et de redoublement déjà mis en scène dans la pièce, quoique l'ethnicité est sans doute fédératrice pour des spectateurs modernes là où le texte élisabéthain n'en a cure. Du point de vue stylistique, les marques d'ethnicité permettent aussi de «hausser le ton», et d'ajouter aux effets formels de la chorégraphie des effets décoratifs attirant l'attention du spectateur sur la mise en scène davantage que sur le contenu des paroles. La cacophonie, ou l'impression de chaos insensé est soulignée dans les deux textes par la prédominance de la forme per se, à savoir de l'audio-vision «réduite» où seule l'intensité est perceptible. Cette intensité gestuelle constituant un langage au premier degré peut s'analyser comme une sorte de discours baroque : bras tendus menaçant de tuer à bout portant, regards fixes, cambrure des reins hispanos et tics nerveux des visages de leurs adversaires. L'exagération baroque est également d'ordre chromatique : monochromie voyante des voitures jaune vif / bleu outremer, polychromie vulgaire des chemises hawaïennes et rouge et noir des tenues hispanos, et enfin auriculaire (la musique semblant émaner d'une voiture s'arrête soudain lorsqu'un personnage fait «chut», créant un fort contraste entre le vacarme et le silence). Toutefois cette «audio-vision réduite» est, dans la pièce, mise au 
service d'une condamnation de la haine entre créatures de Dieu, alors que, dans le film, elle condamne la violence inter-ethnique. La pièce souligne l'aberration de la haine entre frères, le film motive cette haine par l'ethnicité. Ce faisant, le film perd la représentation de l'universel, d'où l'introduction d'un autre effet de motivation, la personnalité de Tybalt qui porte les stigmates du «tueur en série» des films de série $B$ (il baise son arme avant de viser sa victime, choisissant celle-ci presqu'au hasard, comme le suggère le plan d'un enfant dans sa ligne de mire).

On reconnaît enfin dans cette cacophonie les marques de la pluralité sociolectale, ou polyphonie, telle qu'elle a été analysée par Bakhtine. En effet, la voix de l'opinion publique qui selon lui sert de contrepoint aux voix des instances officielles (Big Brother) dans le roman est ici représentée par les médias : le prologue est d'abord dit par une présentatrice de TV dont l'écran se rapproche de nous lentement pour ensuite nous faire entrer dans l'univers de Verona Beach par effacement du cadre et introduction d'effets de caméra bougée d'abord inexplicables puis bientôt diégétisés dès que nous comprenons que nous pourrions nous trouver dans un hélicoptère survolant la ville. Le discours du chef de la police baptisé Prince correspond aux pastiches des instances officielles dont Bakhtine fait un trait caractéristique du roman anglais (Fielding et Dickens par exemple), effet qui caractérise aussi les voix des parents Montague et des parents Capulet. Le spectateur est bel et bien représenté dans le film par l'écran TV, et par l'annonce par la speakerine d'un événement : la guerre que se livrent les clans ennemis, et cela alors même que le prologue de Shakespeare est en effet dit intégralement (sauf les deux dernières lignes). On pourrait même ajouter que l'inversion des deux familles donne la prééminence aux Montague alors que par ailleurs ce sont les «américains» du film (accent de l'acteur jouant Montague, par exemple, plutôt WASP, l'acteur jouant le père de Juliette et l'actrice jouant sa mère se comportant au contraire comme des parvenus à la fête qu'ils organisent).

Dans la première scène de la pièce tout comme dans la première séquence du film, Benvolio parle pour l'opinion publique tandis que les membres des deux clans sont ridiculisés par leur manière affectée de s'imiter l'un l'autre tout en se haïssant, c'est-àdire par les clichés qu'ils utilisent et qui sont des pastiches du discours institutionnalisé par l'usage (tueur en série, par exemple, ou encore Punk). Même l'ethnicité dont il a été question ci-dessus correspond à ce discours des instances officielles. Chez Bakhtine la 
théorie des voix du roman réaliste comme pastiche des Big Brothers de ce monde est fort convaincante ${ }^{8}$. Dans la pièce on en voit tout l'intérêt dramatique puisqu'elle permet d'opposer les voix non seulement d'individus mais de groupes, de chœurs, à la fois microet macro-cosmiques. Dans le film, le pastiche des voix institutionnelles paraît peut-être plus ludique que vraisemblable à un spectateur européen, et sans doute est-ce là la source de cet effet de cacophonie.

Mais l'incipit du film ainsi que d'autres séquences telles que celle de la rencontre des deux adolescents et leur mort comportent une dimension esthétique différente, où la polyphonie tend tout au contraire au lyrisme, suggérant une inspiration empruntée à l'opéra plutôt qu'à la comédie musicale comme nous allons tenter de le montrer maintenant.

\section{De l'opéra au film-opéra}

Outre les timbres de voix et les connotations culturelles analysées ci-dessus, les voix véhiculent aussi des émotions individuelles. Comme l'écrit Michel Chion à propos de l'écoute réduite :

La valeur effective, émotionnelle, physique et esthétique d'un son est liée non seulement à l'explication causale que nous mettons dessus, mais aussi à ses qualités propres de timbre et de texture, à son frémissement. De la même façon que sur le plan visuel, un réalisateur ou un chef-opérateur ont tout à gagner à raffiner leur connaissance de la matière et de la texture visuelles, quand bien même ils ne feraient jamais de films abstraits. $^{9}$

Les voix peuvent donc s'analyser en écoute réduite, et en représentativité émotive (Greimas aurait dit thymique : euphorique $v s$ dysphorique) qui sont susceptibles d'être investies de valeur causale: nous y voyons un indice causal qui serait le sentiment tel que à «cris» nous fournissons une valeur indiciaire : cris de joie, cris de douleurs, à «voix douce» nous trouvons une cause qui peut-être menace secrète $v s$ confession amoureuse, à "voix forte» nous trouvons une cause explicative qui peut être menace et condamnation (le Prince hurlant l'exil de Roméo avec un mégaphone) ou joie des retrouvailles (Roméo accueillant Balthasar 
à Mantoue), et enfin «silence» qui peut être menaçant (Abra et Tybalt dans la première scène) ou amoureux (nuit d'amour entre Roméo et Juliette). L'utilisation de tels effets de voix opposant des contrastes purement sonores et donc maintenant un maximum d'ambivalence naturelle dans la production de ces sons est une des caractéristiques que partagent le mélodrame et l'opéra, deux genres qui sont maintenant reconnus comme ayant présidé à l'origine du cinéma.

Une interprétation Bakhtinienne de l'Opéra pourrait ajouter à ces valeurs thymiques et leurs explications émotives une valeur sociale telle que le chœur et les mouvements de foule chez Moussorgsky ou Verdi représentent l'opinion publique alors que le baryton représente la voix institutionnalisée, ce qui se trouve exprimé dans les traités de composition (voir, pour les instruments Pierre et le Loup bien sûr). Dans le film, l'ambivalence naturelle de ces contrastes est résolue par l'utilisation de cette typologie de l'expressivité (Baz Luhrmann est du reste metteur en scène d'opéra, et danseur d'opéra) empruntée à l'art de l'opéra.

Il devient pertinent dans cette perspective d'analyser une séquence du film où le texte de Shakespeare est utilisé à la manière d'un livret d'opéra, c'est à dire découpé de manière à en faire ressortir certains vers frappants et aisément retenus par le spectateur permettant de mieux s'approprier le film, et remplissant une fonction comparable à celle de l'aria ou air célèbre qui est une des caractéristiques de l'opéra.

Il s'agit de la mort de Mercutio, scène au cours de laquelle la comédie se métamorphose comme par hasard en tragédie.

Le film met en relief la théâtralité de la scène en insistant sur le point de vue du spectateur d'opéra. Mercutio meurt sur scène comme à l'opéra, et le cadrage souligne la rigor mortis par l'angle sur son corps allongé devant nous à l'horizontale, raidi et immobile. Roméo agenouillé à ses côtés se couche sur sa poitrine et la caméra coupe sur un plan en forte plongée, tout en restant toujours en face, de sorte que le point de vue du spectateur se trouve reproduire celui d'un spectateur dans une salle d'opéra ${ }^{10}$ qui serait assis au milieu et à mi-hauteur et non celui d'un spectateur dans une salle de cinéma pour qui tous les angles sont possibles par définition. Le décor sur la plage où se déroule la scène connote le décor entourant une scène d'opéra, décor qui est parfaitement crédible dans cette scène puisque nous l'avons déjà vu lors de la première rencontre avec Roméo (I.1.159-238) où il semble être un ancien lieu de festivités touristiques abandonné et délabré, dont seule la façade tiendrait 
encore debout.

Le texte de la pièce est utilisé à la manière d'un livret d'opéra. En effet, les mots échangés par Benvolio et Mercutio sur la nécessité de quitter les lieux ou de les occuper au contraire (III.1.113), ceux prononcés lors de l'arrivée de Tybalt sur le lieux (33-5) consacrés au défi lancé par Mercutio à Tybalt, ceux par lesquels Roméo exprime son refus de se battre et la bagarre entre Mercutio et Tybalt (61-92), puis la malédiction prononcée par Mercutio avant de mourir (dans la pièce de quitter la scène avec Benvolio), la lutte entre Roméo et Tybalt au cours de laquelle on entend les vers 126-8, et le vers 129 est répété «Either thou or I, or both, must go with him». Tybalt est tué et Roméo tombe à genoux pour dire le vers central : «O, I am fortune's fool». Là aussi la caméra prend de la hauteur de sorte que là encore nous voyons le personnage du point de vue d'un spectateur situé très haut dans un fauteuil d'opéra. Cette technique est aussi utilisée lors du dernier plan sur le suicide de Juliette dans le tombeau. On entend encore quelques mots: «Romeo, away, be gone !» et «Why dost thou stay ?».

Quant aux accessoires de cette scène, ils renvoient également aux codes de l'opéra, mais peut-être davantage encore aux conventions du cinéma. Ainsi en va-t-il par exemple des pistolets remplaçant les épées. On rappellera ici que l'équivalent entre «sword» et l'icône pistolet a été justifié dès la première bagarre à la station d'essence : on a vu que «sword» est la marque de fabrique des pistolets que les garçons utilisent, par un insert sur le pistolet de Benvolio au moment où il dit : «Put up your swords. You know not what you do !» (I.1.63), et un deuxième insert, sur le pistolet dans la voiture de Montague lorsqu'il arrive sur les lieux et descend la vitre fumée de sa limousine noire (façon gangster de Little Casar) pour observer le désastre comme s'il l'avait lui-même savamment orchestré, pistolet sur lequel est gravé «Longsword». L'utilisation de l'icône /voiture/ dans le duel mortel entre Roméo et Tybalt renvoie au motif obligé de tout film d'action : une poursuite en voiture se terminant par un accident; la voiture bleue de Tybalt se retourne et Tybalt en sort en rampant; il se redresse pour faire face à un Roméo métamorphosé en bête furieuse non seulement par les yeux écarquillés et les narines dilatées de l'acteur mais aussi par un cadrage très serré qui évoque les conventions des bandes dessinées. Roméo l'abat et il tombe à la renverse dans de l'eau noire (la scène est devenue nocturne au cours de la poursuite). La violence de la poursuite et la métamorphose soudaine du gentil adolescent en monstre furieux servent de «climax» émotionnel, de sorte que le 
plan sur Roméo agenouillé et s'écriant les yeux levés vers nous / le ciel : «O, I am fortune's fool», amorcé par un battement de cils sur le plan précédent et orchestré par un silence brutal faisant suite à la chute de Tybalt à la renverse, se charge de connotations bibliques telles que le péché originel et la découverte du mal. Cet effet d'opéra est toutefois banalisé par le plan suivant cadrant la statue immense du Christ dont nous savons qu'il veille sur la ville en compagnie de l'hélicoptère de la gendarmerie, soulignant le message religieux de sorte que les codes de l'opéra deviennent plutôt des procédés rhétoriques que des signes explicites. Autrement dit nous sommes à l'opéra sans le savoir, et peut-être surtout devant un film-opéra.

Le cadrage et le montage, codes du cinéma s'il en est, nous donnent en effet surtout l'impression d'être au cinéma lorsque nous visionnons cette version de la pièce élisabéthaine. En ce qui concerne le cadrage, en particulier, outre les clichés de la folle course poursuite en voiture, nous reconnaissons des effets citationnels en particulier au célèbre Rebel Without a Cause de Nicholas Ray (La Fureur de Vivre (1955)) qui révéla James Dean au monde des cinéphiles. L'angle sur les deux garçons lorsqu'ils s'apprêtent à s'affronter pour la dernière fois et celui par lequel Nicholas Ray ouvre son film, cadrant James Dean en haut de l'écran, allongé sur l'asphalte, tendant la main vers un jouet au premier plan. L'effet citationnel comporte en outre un effet d'ironie tragique puisque le jouet dans ce plan cadré lui aussi au ras de l'asphalte ruisselant de pluie est le pistolet fatidique vers lequel les deux garçons tendent la main, et dont Roméo s'empare le premier. Le haut de l'écran figure Roméo, le bas figurant Tybalt, et le pistolet sur l'asphalte entre eux est au centre de l'écran.

Quant au montage, il comporte certaines caractéristiques qui méritent d'être soulignées, en particulier l'utilisation du syntagme alterné. Par exemple, les vers de Shakespeare représentant la pensée de Roméo pour Juliette avant de se transformer en justicier (113-5 : «O sweet Juliet, / Thy beauty hath made me effeminate / And in my temper softened valour's steel !») trouve son équivalent par un plan sur Juliette heureuse de l'attendre (III.2.20-8: «Come gentle night...»). Ce plan est raccordé sur celui de Roméo chargeant la voiture de Tybalt. Par ailleurs, au moment où Roméo profère ses menaces de mort («Mercutio's soul / Is but a little way above our heads / Staying for thine to keep him company. / Either thou or I, or both, must go with him», III.1.126-9), la caméra cadre un plan serré sur le visage de Tybalt en ralenti annonçant sa mort, raccord sur un 
plan très rapide de Juliette. C'est-à-dire que la scène 2 de l'acte III est divisée en fragments et que ceux-ci sont montés en syntagmes alternés avec la scène de la bagarre : un effet de contrepoint est donc obtenu par ce montage suggérant la simultanéité des deux actions, tout en renforçant la tragédie en montrant à l'avance ses conséquences sur la destinée de Juliette, victime innocente. Il ne s'agit pas seulement d'être fidèle au texte de Shakespeare en figurant par le montage le monologue intérieur de Roméo seul sur scène à cet instant et représenté par conséquent comme un véritable héros tragique responsable en partie de sa fin tragique. En effet, la pensée de Juliette joue le rôle de l'avertissement au héros par la sibylle, avertissement qu'il entend, certes, mais qu'il interprète comme une incitation au combat, alors qu'il aurait dû le comprendre comme une incitation à l'exercice de la justice civique. Il s'agit de renforcer par le parallèle entre, d'une part notre vision du héros pacifique engagé par son mariage sur la voie de la paix, et, d'autre part, sa métamorphose funeste en justicier vengeur. Le reste de la scène consacrée à l'attente de Juliette, à savoir l'arrivée de sa nounou et l'annonce de la mort de Tybalt est monté après une séquence représentant la condamnation à l'exil, sentence sans appel prononcée par le Prince / policier, et celle de Roméo réfugié chez Friar Laurence et ses projets : «But look thou stay not till the Watch be set...» (III.3.148-54). Les pleurs de Juliette à l'annonce de la mort de Tybalt sont donc donnés une fois encore en montage alterné. Ceci permet un effet de surprise lorsque sur les pleurs de Juliette est monté un plan de Roméo, puis un plan de Juliette se retournant, son visage en larmes se métamorphosant en un visage de bonheur, les larmes devenant par ce contexte leur contraire, à savoir des larmes de joie.

On pourrait aller jusqu'à suggérer que non seulement le film transforme la pièce en opéra mais la transforme aussi en mélodrame. Le mélodrame est à distinguer de l'opéra puisqu'il s'agit avant tout d'un art parallèle au théâtre et fondé sur le silence. Le lien entre le cinéma muet et le mélodrame demande à être exploré, ce qui n'a pas lieu d'être $\mathrm{ici}^{11}$. Cependant, nous étudierons la dernière séquence du film où l'opéra et le mélodrame viennent renforcer les codes cinématographiques, ceux-ci étant eux-mêmes également nourris d'effets spéciaux.

\section{Le souffle shakespearien}

Si le texte de Shakespeare est réduit à un strict minimum, il est cité fidèlement et non dénaturé. C'est le cas en particulier pour 
la scène du tombeau dont nous dirons ici quelques mots. Cette scène est exemplaire à plus d'un titre mais sans doute l'effet le plus souvent cité est celui du baiser sur les lèvres et la métaphore de l'union des âmes dans la vie éternelle après la mort que représente l'échange du souffle de deux vies.

Au cinéma comme à l'opéra, cette scène pose le problème du silence de la mort et celui de la voix indispensable pour éveiller l'émotion chez le spectateur ${ }^{12}$. L'alternance des deux morts, celle supposée de Juliette puis celle réelle de Roméo permet au spectateur d'entendre deux solos alors même que la voix de Roméo est celle d'un très jeune garçon dont on accepterait presque qu'il n'ait pas encore fini de se muer en voix adulte, et celle de Juliette est également très jeune, presque mal assurée (chez les Élisabéthains, une autre voix de jeune garçon avant la mue, et de ce fait très proche de celle de Roméo). Le texte du film est le suivant ${ }^{13}$ :

\section{Rom}

The music stops : absolute silence.

Juliet,

Why art thou yet so fair? Shall I believe

That unsubstantial death is amorous,

[...] (looks above) keeps

Thee here in the dark to be his paramour? (pauses)

For fear of that I still will stay with thee

And never from this palace of dim night

Depart again. Here, here will I remain

With worms that are thy chambermaids. (Puts the ring and chain on her finger; kisses her; wipes his eyes, breathes hard, crying; pauses then lies down beside her.) $\mathrm{O}$ here

Will I set up my everlasting rest

And shake the yoke of inauspicious stars

From this world-wearied flesh. Eyes, look your last!

Arms, take your last embrace ! and lips, $O$ you

The doors of breath, seal with a righteous kiss (drinks the poison, eyes lifted to heaven, not seeing what we see, that Juliet's eyes are now open and her fingers move slightly)

A dateless bargain to engrossing death! (looks at Juliet's now wide open eyes and falls shivering beside her)

Jul. What's here? ...

Poison, ...

.... drunk all, and left no friendly drop 
To help me after ? I will kiss thy lips. (161 65)

Haply some poison yet doth hang on them (she kisses him; the camera cuts to Romeo looking at her)

Rom. Thus with a kiss I die (120) (turns his head to the left and dies)

(Juliet sits up on her death bed and looks around; utters a heart-rending groan, snatches Romeo's gun / dagger, - acousmatic ${ }^{14}$ sounds of klicking gun in otherwise complete silence - raises her eyes to heaven and puts the gun to her temple: the loud report of a gun is heard as the camera cuts to a downward on the two bodies: she falls and the magic pathway that led Romeo from the door to her bed is now framed to rise to the top of the screen, suggesting a magic Milky Way leading up to heaven).

Le silence qui sert de toile de fond à ces vers les rend particulièrement audibles et attire notre attention sur la métaphore du souffle, souffle de l'âme matérialisée dans cette scène par le souffle du corps de l'acteur présent sur la scène et rendu à l'opéra par le chant du cygne des héros ou héroïnes devant mourir sur scène. Le rôle du «O» dans le texte, «O here I will rest», «O you the doors of breath» symbolisant la quintessence de l'âme emprisonnée dans le corps : «eyes, look», «arms, embrace», «lips», est souligné par le montage et le décor.

Le décor est d'abord une colonne scintillante au centre de la lourde porte entrebâillée, puis l'œil de Roméo y est cadré en contrechamp afin de suggérer un effet de caméra subjective ${ }^{15}$ lorsqu'avec le plan suivant nous découvrons le décor de l'intérieur de l'église, rendu plausible par le souvenir qui est aussitôt évoqué : c'est le rêve de Roméo avant le bal au début du film, mais rendu surnaturel par la magie de milliers de bougies «hispanos» éclairant l'intérieur obscur de l'église. Le chemin tracé devant lui est féerique (effets spéciaux soulignant explicitement la rupture avec le monde réel) et connote l'entrée dans la vie après la mort. Cette voie magique et immatérielle devient la Voie Lactée, le chemin cosmique des bandes dessinées, certes, mais transformé par le texte poétique en poésie de plein droit.

Le montage y est aussi pour beaucoup : les deux amants sont cadrés très serrés, comme coupés du monde, monde dont le silence qui s'est installé (nous entendons les sons acousmatiques s'éteindre 
peu à peu lorsque Roméo ouvre la porte), et leurs lèvres sont très proches l'une de l'autre, en particulier lorsque Roméo prononce le vers qui résume toute l'action: «Thus with a kiss I die». On touche donc ici à la question de la synchronie, effet déjà rendu aisément repérable dans l'ouverture du film connotant une Ouverture d'Opéra, où la synchronisation du son-off (musique de fosse) avec le cadrage sur la ville est d'autant plus marquée qu'elle s'inscrit dans un mouvement d'hélicoptère imprévisible et soumis à des changements brutaux. C'est le contraste entre les deux rythmes, l'un scandé par l'orchestre, l'autre désordonné et aléatoire, et leur coïncidence soudaine en points de synchronie créant un effet de synchrèse ${ }^{16}$ inattendu qui rend cette ouverture particulièrement saisissante (échos du début d'Apocalypse Now en plus, sans doute).

Dans le dialogue des amants, on observe aussi des effets de synchrèse entre le texte et le cadrage, comme les remarques cidessus le suggèrent, qui contribuent fortement à l'impact émotionnel sur le spectateur. Toutefois, ces effets de synchrèse entre les vers et les images ont aussi pour effet de souligner les vers eux-mêmes, leur étrangeté dans un dialogue de la fin du vingtième siècle et leur beauté aussi. Il y a donc une mise en scène de l'art poétique pour lui-même, nous invitant en quelque sorte à une forme d'écoute réduite des sons per se, analogue en cela au chant d'opéra, et qui a pour effet de souligner la mise en scène de la voix du poète indépendamment de la situation. Le film tire une partie de ses effets d'une représentation de la star Shakespeare comme s'il s'agissait d'un prince des poètes devenu personnage, comme nous allons tenter de le prouver.

Après le coup de feu, on entend les derniers accents d'Iseult dans l'opéra de Wagner, tandis que les amants sur le lit sont cadrés en plongée mais n'occupant que la partie haute de l'écran ${ }^{17}$ et paraissent se déplacer dans les airs (effets spéciaux) au-dessus des bougies «hispanos» qui deviennent ainsi des étoiles cosmiques. Notre description se veut un relevé fidèle de l'effet sur le spectateur de cette avant-dernière séquence du film. la dernière est celle du discours de «Big Brother» prononcé par le policier Prince ; puis la télévision du prologue réapparaît et la speakerine donne les derniers vers. L'effet est saisissant : on croit entendre la voix de William Shakespeare.

Les vers de Shakespeare sont en effet utilisés à la fin comme au début de manière non diégétique, ce qui est certes la fonction du prologue, mais qui, dans la pièce, est dévolue au Prince en guise d'épilogue. 
Ces deux textes et la télévision par laquelle ils sont présentés au spectateur ont certes le rôle énonciatif de la voix de l'opinion publique. Mais cette instance énonciative qui leur vient du prologue et de l'épilogue de théâtre se double d'une deuxième voix ici, la voix du poète. Ainsi, le «récit cadre» dont l'énonciateur appartient au temps présent du spectateur tout en lui rappelant qu'il assiste à un spectacle se déroulant dans un autre temps que ce présent «dramatique» permet également la mise en scène de la voix du poète, dont le double statut - à la fois tourné vers son sujet et s'adressant par le vers directement à son lecteur - fonde tout discours poétique. En donnant ce rôle à la speakerine du journal télévisé, le film rend présent dans notre temps en dehors du temps de la fiction un personnage créé pour les besoins de la fiction. Il $s^{\prime}$ agit de mettre en scène un personnage historique s'adressant à nous du fond des âges, un conteur qui occupe le devant de la scène, et plus crédible pour nous s'il est figuré par le rôle de la speakerine du journal télévisé que si nous étions face à face avec un portrait de Shakespeare lui-même, car plus probable.
A glooming peace this morning with it brings.
The sun for sorrow will not show his head.
Go hence, to have more talk of these sad things.
Some shall be pardoned, and some punishèd.
For never was a story of more woe
Than this of Juliet and her Romeo. (V.3.305-10)
(La télévision s'éteint et le cadre où brillent les milliers de points blancs et gris diminue jusqu'à disparaître tout à fait.)

Cette mise en scène du personnage historique contribue à la crédibilité des paroles, et ratifie l'exactitude de celles-ci ; en effet, s'il en manque beaucoup, et certaines sont répétées plusieurs fois, ou passées sous silence (mais incluses si la syntaxe le permet, comme par exemple V.3.161-2, citées ci-dessus), il semble bien qu'aucune parole prononcée par les personnages dans le film ne soit pas une parole tirée du texte. C'est en ce sens qu'il y a en effet lieu de parler d'authenticité, et donc d'une source d'énonciation unique, impliquant la présence effective de l'auteur personnage historique dans le texte poétique.

La métaphore du souffle figurant l'âme et la vie éternelle qui préside à l'échange entre les amants se double donc d'une représentation du souffle lyrique du poète dans cette dernière 
séquence. La pièce joue déjà sur cette ambiguïté puisque Roméo et Juliette sont eux-mêmes poètes, comme le signale d'entrée de jeu leur rencontre et le sonnet qu'ils improvisent. Les vers qu'ils disent en mourant sont donc rendus plausibles - motivés - par leur personnage; toutefois, en créant un personnage William Shakespeare, le film souligne l'effet de présence du poète-auteur qui est déjà implicite dans la pièce, et joue sur l'effet d'authenticité traditionnellement associé au cinéma. La caméra a en effet hérité de l'appareil photographique une valeur documentaire et un fort effet de réel, et sans doute le fait que la voix de William Shakespeare soit en quelque sorte toujours en son hors-champ ${ }^{18}$, c'est-à-dire à la fois dans la diégèse et dans le hors-cadre où se trouve le spectateur dans le noir contribue fortement à l'illusion d'entendre William Shakespeare lui-même.

\section{Conclusion}

Le Roméo et Juliette de Baz Luhrmann est donc une représentation de William Shakespeare lui-même, confirmant ainsi le titre anglais : William Shakespeare's Romeo + Juliet, et mettant l'accent sur la star Shakespeare. Le film réussit la gageure de faire dire et de faire écouter des vers du seizième siècle à des adolescents n'ayant sans doute jamais entendu cette musique auparavant, et, ce faisant, le film rend aux vers leur fonction première: faire pénétrer par la beauté sonore du langage le spectateur dans un univers insoupçonné de lui, inconnu, aussi palpitant qu'un univers de science-fiction ou un univers fantastique, mais également doté de tous les stigmates d'un réel historique, aussi émouvant que la confrontation brutale avec l'épave réelle du Titanic dans la version récente de ce récit. Tout comme les profondeurs insondables d'où le passé surgit et se métamorphose dans ce film en réalité palpable, le fond noir de William Shakespeare's Romeo + Juliet d'où émerge l'écran de la télévision signifie un réel non seulement polyphonique mais aussi polymorphe. Il s'agit en effet à la fois de l'émergence sur notre écran d'une image particulière dans le présent de la projection filmique, et d'une représentation métaphorique des voix de la nuit: nuit des temps et nuit de l'inconscient s'y fondent en une seule spirale dont le pouvoir rhétorique a fait l'objet de commentaires enflammés dès la naissance du cinéma. Or la «réalité» qui émerge de ce fond noir ou qui s'y évanouit à la fin du film a le pouvoir d'être en quelque sorte deux fois réel, puisqu'il s'agit à la fois du vers effectivement dit par des acteurs élisabéthains il y a quatre siècles et qui nous 
parviennent sous une forme inchangée, et du souffle de cet acteur incarnant le souffle de l'inspiration poétique. Telle est sans doute, du moins en partie, la nature de la fascination qu'exerce sur le spectateur l'authenticité des vers prononcés par les personnages du film.

Raphaëlle COSTA DE BEA UREGARD

Université de Toulouse-Le Mirail

\section{NOT E S}

${ }^{1}$ Baz Luhrmann, réalisateur australien connu pour un premier film Ballroom Dancing (Festival de Cannes 1992). Titre du film: William. Shakespeare's Romeo + Juliet. Scénario : Craig Pearce, Baz Luhrmann d'après William Shakespeare. Image : Donald M. MacAlpine. Musique : Nellee Hooper, Craig Armstrong, Marius de Vries. Producteur : Bazmark Production, Gabriella Martinelli, Baz Luhrmann. Avec Leonardo DiCaprio (Roméo), Claire Danes (Juliette), Brian Dennehy (Montague), Paul Sorvino (Capulet), Harold Perrineau (Mercutio), John Leguizamo (Tybalt). 120 minutes. États-Unis, 1996. Disponible en V.O. VHS/Pal-Secam en G.B., et en V.F. en France.

${ }^{2}$ Précédentes adaptations : Romeo und Julia im Schnee (1920), Ernst Lubitsch, avec Gustav von Wangenheim et Lotte Neumann; Les Amunts de Vérone (1948), André Cayatte avec Serge Reggiani et Martine Carol; Roméo et Juliette (1954), Renato Castellani ; West Side Story (1961), Robert Wise avec Nathalie Wood et Richard Beymer; Roméo et Juliette (1968), Franco Zeffirelli avec Leonard Whiting et Olivia Hussey; China Girl (1987), Abel Ferrara; Pocahontas (1995), Mike Gabriel et Eric Goldberg avec les effigies de Tom Cruise et Naomi Campbell. Ce recensement est paru dans Première, Mai 1997. Penny Starfield, «L'effet libérateur ou inhibiteur de Romeo and Juliet : réflexions sur Shakespeare au cinéma», Ruth Morse (ed.), Shakespeare - Préjugés et Tolérance Cahiers Charles V, $\mathrm{n}^{\circ} 24,1998$, p. 197-212, signale aussi Romeo and Juliet de George Cukor (1935) et Tromeo and Juliet de Lloyd Kaufman (1996).

${ }^{3}$ «Ma grande fierté, c'est qu'avec Roméo et Juliette nous ayons battu, le premier week-end de sortie au box-office américain, Stallone et son Daylight, et que la bande originale du film soit trois fois disque de platine aux U.S.A.», déclare le réalisateur dans une interview accordée à Michel 
Rebichon : «Baz Luhrmann : rock'n'roll attitude», Studio, avril 1997, p. 79.

${ }^{4}$ José Arroyo, «Kiss-Kiss - Bang-Bang», Sight and Sound, March 1997, p. 6-9. Nous remercions ici Sandy Bazckowski pour sa contribution à notre recherche dans le cadre de sa maitrise sur ce film, et pour avoir attiré notre attention sur son intérêt.

${ }^{5}$ Arroyo, Sight : «William Shakespeare's Romeo \& Juliet is a kiss-kiss bang-bang movie. It's got action, spectacle and romance and it aims to entertain».

${ }^{6}$ Michel Chion, L'Audio-Vision - Son et image au cinéma, Paris, Nathan, 1990. Chion distingue trois attitudes d'écoute chez le spectateur: 1) l'écoute causale, lorsque le son renseigne sur sa cause. Soit la cause est visible soit elle ne l'est pas, mais identifiée par une supputation logique. C'est sur ce savoir que brode l'écoute causale qui ne part pas de zéro ; 2 ) l'écoute sémantique, celle qui se réfère à un code ou à un langage pour interpréter un message, le langage parlé, le morse ; 3) l'écoute réduite, appellation due à Pierre Schaeffer est l'écoute qui se porte sur les qualités et les formes propres au son, indépendamment de sa cause et de son sens. Le son est pris comme objet d'observation.

${ }^{7}$ Le syntagme alterné ne doit pas être confondu avec le syntagme parallèle, même si l'anglais utilise le même terme, la distinction est de taille : le syntagme alterné est d'ordre diégétique, événementiel, et montre deux actions se déroulant dans le même temps dans deux lieux différents; le syntagme parallèle est métaphorique : le montage de plans successifs sans lien chronologique, entretenant seulement un rapport thématique. André Gardies et Jean Bessalel, 200 mots-clés de la théorie du cinéma, Paris, Éditions du Cerf, 1998 et Frank Beaver, Dictionary of Film Terms The Aesthetic Companion to Film Analysis, New York, Twayne Publishers, 1994.

${ }^{8}$ Mikhaïl Bakhtine, Esthétique et théorie du roman (1975), Paris, Gallimard, 1978, p. 122-51, «Le plurilinguisme dans le roman».

${ }^{9}$ Chion, Audio-vision, p. 30.

${ }^{10}$ Voir en particulier l'utilisation des points de vue divers du spectateur dans une salle d'opéra par Losey pour la prise de vue de Don Giovanni.

${ }^{11}$ Voir par exemple Peter Brooks, The Melodramatic Imagination Balzac, Henry James, Melodrama and the Mode of Excess, New York, Columbia University Press, 1985.

${ }^{12}$ Une comparaison entre cette scène et celle filmée par la réalisatrice australienne, Jane Campion, pour le baiser d'adieux entre Ralph et Isabel dans Portrait of a Lady serait sans doute fort éclairante, d'autant plus qu'il semblerait que les deux réalisateurs ont fait leurs études à la même école cinématographique et ensemble.

${ }^{13}$ Les italiques sont les miennes n'ayant pas pu disposer du scénario, et de toutes façons en sachant que le scénario n'est jamais la même chose que le texte final. 
${ }^{14}$ Chion distingue une zone sonore visualisée : le son-in, où la cause du son est visible, et deux zones non-visualisées qu'il appelle zones acousmatiques : le hors-champ (voice-over) et le son-off (background score). Chion, L'audio-vision, p. 65-6.

${ }^{15}$ François Jost, L'Eil-caméra, Lyon, P.U.L., 1985 ; voir aussi du même auteur Le Temps d'un Regard, Méridiens-Klincksieck, 1997.

${ }^{16}$ Chion, L'audio-vision, p. 55 : «La synchrèse est la soudure irrésistible et spontanée qui se produit entre un phénomène sonore et un phénomène visuel ponctuel lorsque ceux-ci tombent en même temps, cela indépendamment de toute logique rationnelle».

${ }^{17}$ On pense ici à une aquarelle célèbre de Rossetti représentant des amants enlacés dans la mort paraissant flotter en haut du cadre sur un fond d'étoiles filantes: Paolo and Francesa; le même effet a été utilisé par Chagall.

${ }^{18}$ Voir note 14 ci-dessus. 